\title{
Peritoneal Fluid
}

National Cancer Institute

\section{Source}

National Cancer Institute. Peritoneal Fluid. NCI Thesaurus. Code C77612.

The small amount of fluid that is generated in the abdominal cavity to lubricate the peritoneum. 